\title{
A study of new manganese complexes as potential driers for alkyd paints
}

\author{
E. Bouwman, R. van Gorkum
}

\begin{abstract}
In the search for new autoxidation catalysts, the oxidative crosslinking of ethyl linoleate (EL) has been monitored using time-resolved FT-IR (Fourier transform IR spectroscopy), size-exclusion chromatography and GC/MS (gas chromotography/mass spectroscopy). These methods seem to be quite sensitive to changes in the structure of the catalyst. It has been found that the compound $\left[\mathrm{Mn}(\mathrm{acac})_{3}\right](\mathrm{Hacac}=2,4-$ pentanedione) is an efficient catalyst for the oxidation and the oligomerization of EL, which is proposed to proceed not only via hydroperoxide decomposition, but also through substrate activation. The system $\left[\mathrm{Mn}(\mathrm{acac})_{3}\right]$ with added bpy $\left(=2,2^{\prime}\right.$-bipyridine $)$ has a very high activity for the oxidation of EL. In situ formation of the species $\left[\mathrm{Mn}^{\mathrm{II}}(\mathrm{acac})_{2}(\mathrm{bpy})\right]$ and $\left[\mathrm{Mn}^{\mathrm{III}}(\mathrm{acac})_{2}(\mathrm{bpy})\right]^{+}$, and the high reactivities of these two species with hydroperoxides and with EL, respectively, are proposed as an explanation for the observed high oxidation rate.
\end{abstract}

Keywords Alkyd paint, Cobalt-based catalysts, Manganese derivatives, Cobalt derivatives, Drier catalyst, Ethyl linoleate, Bipyridine

\section{Introduction $^{1}$}

Alkyd paints are based on alkyd resin precursors, which are polyesters incorporating unsaturated fatty

Presented at XXVIIIth FATIPEC Congress, organized jointly by the Hungarian Chemical Society (MKE) and the Polish Association of Chemical Engineers (SITPCHEM), in Budapest, Hungary, June 12-14, 2006.

E. Bouwman $(\bowtie)$, R. van Gorkum

Leiden Institute of Chemistry, Leiden University, P.O. Box

9502, Leiden 2300 RA, The Netherlands

e-mail: bouwman@chem.leidenuniv.nl acid chains such as oleic acid, linoleic acid, linolenic acid, and ricinoleic acid. In the drying process of a paint film, the solvent evaporates (physical drying), and then chemical reactions take place, resulting in the curing of the binder.

The chemical 'drying' of alkyd paints is a metalcatalyzed, oxidative crosslink-forming process, mainly based on the autoxidation of the unsaturated fatty acid side chains. The autoxidation of the diene moiety, leading to a dry paint film is illustrated, in a greatly simplified form, in Fig. 1. The reaction is initiated by hydrogen abstraction of the doubly activated methylene group. The resulting radical, $\mathrm{R}^{\bullet}$, reacts with dioxygen, according to the well-known autoxidation mechanism, leading to hydroperoxide species $(\mathrm{ROOH})$. These hydroperoxides decompose in a metal-catalyzed reaction to alkoxyl $\left(\mathrm{RO}^{\bullet}\right)$ and peroxyl radicals $\left(\mathrm{ROO}^{\circ}\right)$. Through the recombination of the radicals, a three-dimensional polymer is formed that is responsible for the hardening of the paint.

Among the environmental challenges arising in the last decade, that concerning the substitution of suspected toxic components in common organic coating materials is of great importance. The search for acceptable alternatives to toxic chemical additives in alkyd paints, especially the antiskinning agents and the cobalt drier catalysts, has recently become a subject of intense industrial and academic interest. ${ }^{1-8}$

The most important and recognized role of drying agents in the paint formulations is to enhance the decomposition of the relatively stable hydroperoxides. The role of the metal ion in the first initiation step is still an issue of debate. The most widely used driers for solventborne paints contain cobalt 2-ethylhexanoate as the primary drier. To screen and to compare a large number of metal complexes for their potential use as a drying catalyst, a fast, simple, and reliable test system has been developed. First of all, the performance of the commercial cobalt driers and manganese driers were 


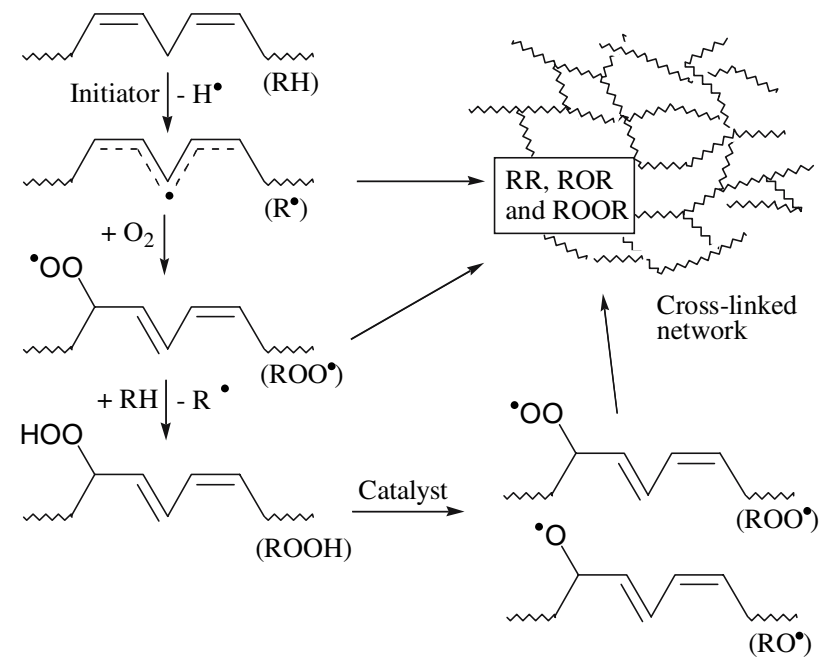

Fig. 1: Schematic impression of paint drying: the catalyst decomposes hydroperoxides to form alkoxyl and peroxyl radicals. Termination reactions create the crosslinked network

analyzed; the effect of bipyridine inclusion on the reactivity of these driers has been investigated and the results compared with the actual effectiveness of these systems in real alkyd paints. ${ }^{3}$ In later stages, new drier catalysts based on manganese were developed. ${ }^{2,7}$ Also, the effect of the antiskinning agent, methyl ethyl ketoxime (MEKO), on the activity of a drier has been monitored using this test system. ${ }^{6}$

\section{Development of the test system}

\section{Modeling alkyd paint}

In order to avoid using complex paint mixtures, compounds can be used that model certain aspects of the mixture. Since, in alkyd paint drying, most of the chemistry takes place at the unsaturated fatty acid tails of the binder compound, the entire binder resin can be modeled by using compounds that structurally or chemically resemble this fatty acid chain. Dienes such as 4,7-heptadiene, ${ }^{9} 3,6$-nonadiene, ${ }^{10}$ and 2,5-undecadiene, ${ }^{11}$ as well as methyl and ethyl esters of the unsaturated fatty acids linoleic, linolenic, and ricinoleic acid, ${ }^{12-15}$ have been used as model compounds for alkyd resin precursors. An advantage of this approach, besides having a much simpler system to study, is that when model compound mixtures become 'dried', they remain liquid and consequently are more easily studied using standard analytical techniques. In the present study, ethyl linoleate (EL, see Fig. 2), the ethyl ester of the fatty acid linoleic acid, was used. A wide range of analytical techniques has been used to study the autoxidation of alkyd paint model compounds. These techniques include ${ }^{13} \mathrm{C}-\mathrm{NMR},{ }^{10-13} \mathrm{GC},{ }^{13}$ HPLC, ${ }^{9-11}$ FT-IR, ${ }^{14,16-18}$ SEC, ${ }^{12,13}$ and SIMS. ${ }^{15}$

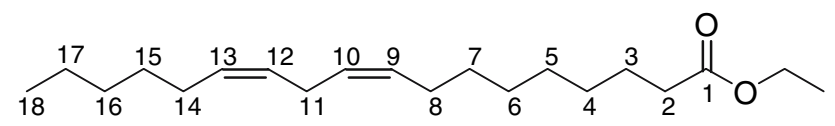

Fig. 2: Ethyl ester of (Z,Z)-9,12-octadecadienoic acid; ethyl linoleate

To screen different metal complexes quickly for their ability to enhance the 'drying' of EL, the two more useful parameters to monitor are the rate of the autoxidation reaction and the extent of the crosslinking that takes place. Time-resolved Fourier transform infrared spectroscopy (FT-IR) has been used to study the kinetics of EL oxidation. Size exclusion chromatography (SEC) has been used to measure the extent of crosslinking.

The experimental procedures that were used in the studies can be found in the original research papers. $^{3,7,19}$

\section{Time-resolved FT-IR experiments}

The kinetics of fatty acid oxidation can be studied by time-resolved FT-IR spectroscopy., ${ }^{2,3}$ The cis $\mathrm{H}-\mathrm{C}=\mathrm{CH}$ stretching vibration at $3010 \mathrm{~cm}^{-1}$ is especially suitable for following the disappearance of the substrate in time. ${ }^{20}$ In the first step of the reaction, a hydrogen atom is abstracted from the reactive methylene group of the cis,cis-1,4-diene moiety. After the rearrangement of the resulting pentadienyl radical and reaction with dioxygen, a hydroperoxide is formed. One of the cis $\mathrm{H}-\mathrm{C}=\mathrm{CH}$ hydrogens has become a hydrogen atom on a secondary carbon atom (see Fig. 3). The decrease of the IR vibration due to the disappearance of this cis $\mathrm{H}-\mathrm{C}=\mathrm{CH}$ hydrogen atom can thus be associated directly with the first step of the autoxidation reaction.

To monitor the autoxidation of EL in time, an FT-IR spectrum is recorded (automatically) every 5 min. Figure 4 shows (part of) several representative spectra of an oxidation experiment. For each spectrum, integration is carried out for the area between 2992 and $3025 \mathrm{~cm}^{-1}$. As a result, a table is obtained with the area of the $3010 \mathrm{~cm}^{-1}$ peak at different times. The logarithmic plot of these data gives a straight line and thus, as a first approximation, the oxidation of EL follows pseudo first-order kinetics. From the slope of this graph, the first-order reaction rate of $\mathrm{H}^{\bullet}$ radical abstraction can be determined. In some cases, an induction period occurs. This can vary from several minutes to many hours. The occurrence of an induction time can have several explanations. However, the more important factors are the concentration of hydroperoxides in EL at the start of an autoxidation reaction (see the later section headed 'Determination of the hydroperoxide concentration in ethyl linoleate') relative to the catalyst concentration and the metal complex that is used as a drying catalyst. 


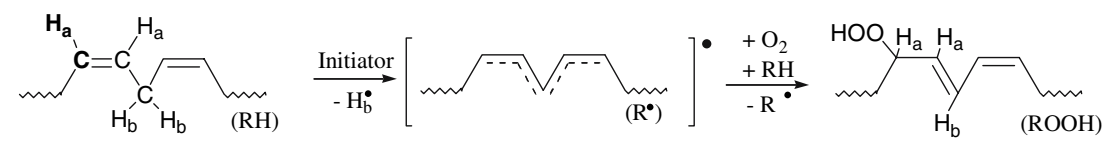

Fig. 3: The cis $\mathrm{H}_{\mathrm{a}}-\mathrm{C}=\mathrm{CH}$ hydrogen of a 1,4-pentadiene unit in a fatty acid disappears in the first step of the autoxidation due to abstraction of $\mathrm{a} \mathrm{H}_{\mathrm{b}}$ atom and rearrangement of the resulting pentadienyl radical

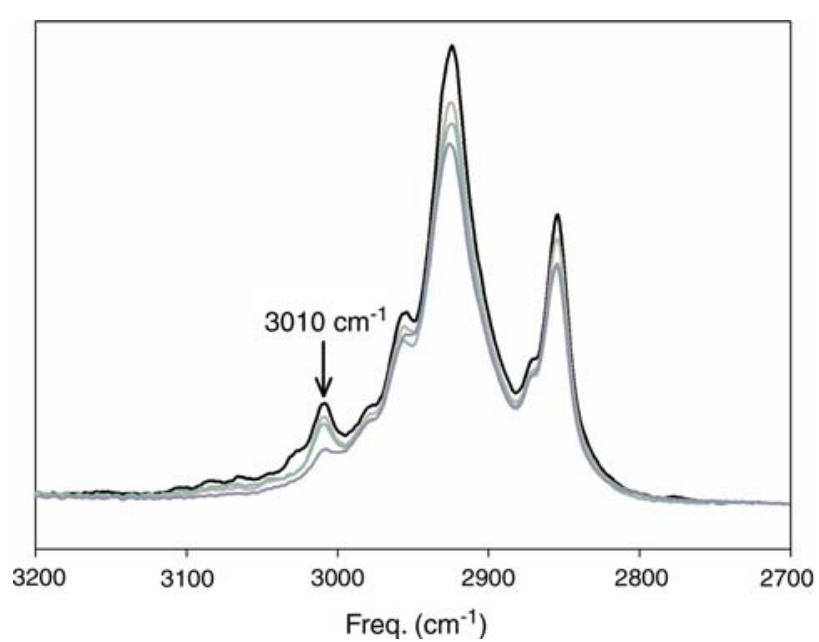

Fig. 4: $2700-3200 \mathrm{~cm}^{-1}$ region of the FT-IR spectra of EL at increasing reaction times (curves at $0,50,100$, and $750 \mathrm{~min}$ are shown) during an autoxidation reaction. The peak at $3010 \mathrm{~cm}^{-1}$ decreases as the autoxidation reaction proceeds

Since the metal-catalyzed autoxidation of EL is a radical reaction, an optimal catalyst concentration is expected because with higher concentrations saturation would occur. To determine the optimal concentration for the screening of different metal catalysts, a series of measurements with different concentrations of cobalt 2-ethylhexanoate (Co-EH) has been performed. The curve, as obtained from a plot of the reaction rate constants for different molar ratios $\mathrm{Co} / \mathrm{EL}$, indeed shows saturation behavior. Using cobalt concentrations in a molar ratio of $\mathrm{Co} / \mathrm{EL}$ higher than $4 \times 10^{-3}$ does not result in a significant increase in the reaction rate, as shown in Fig. 5. Therefore, for all of the FT-IR experiments, a molar catalyst/EL ratio of $2.5 \times 10^{-3}(\mathrm{EL} /$ catalyst $=400 / 1)$ has been used. At this ratio, the reaction rate is still dependent on the metal concentration, but it is not too sensitive to small experimental errors compared with the sensitivity that assesses the very low metal concentrations.

\section{Size exclusion chromatography}

In the metal-catalyzed autoxidation of EL, crosslinks are formed, predominantly because of radical termination reactions. These crosslinks consist of ether bonds, peroxy bonds, and carbon-carbon bonds. ${ }^{13-15}$ Due to crosslink formation, dimers, trimers and even oligomers of EL are formed while the autoxidation

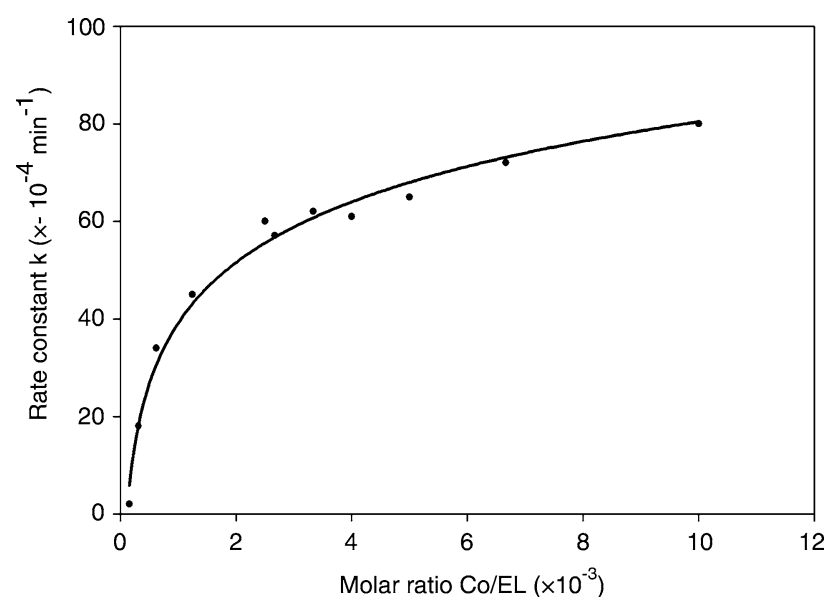

Fig. 5: The effect of the molar Co/EL ratio on the reaction rate

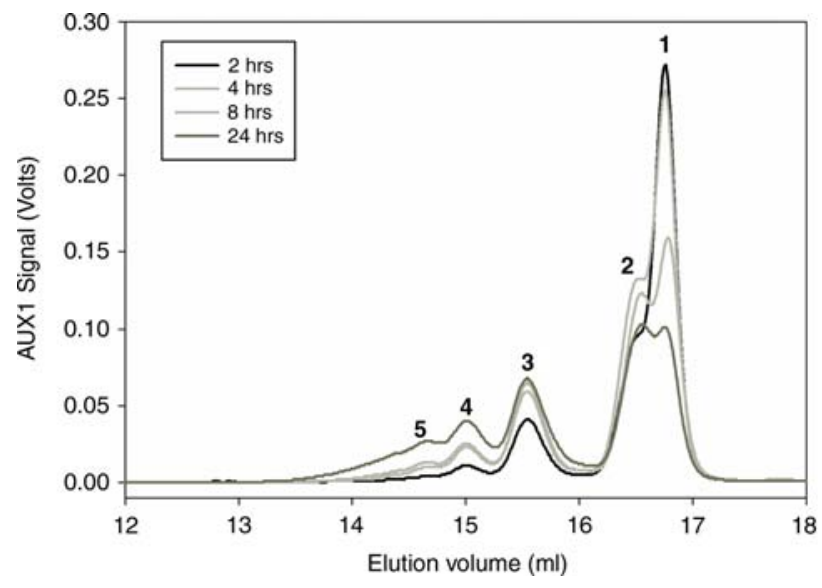

Fig. 6: Several size exclusion chromatograms; samples taken after $2,4,8$, and $24 \mathrm{~h}$ reaction time of an autoxidation reaction mixture of EL with a metal complex drying catalyst. Peak assignments: 1: EL 2: EL-hydroperoxide 3: dimers 4: trimers 5: oligomers

reaction proceeds. Taking samples of an autoxidation reaction mixture at increasing reaction times, and measuring them using SEC gives some insight in to the rate of formation of the different crosslinked species. In Fig. 6 several chromatograms are shown that have been obtained at different times during an autoxidation reaction. The EL peak decreases in time as ELhydroperoxides are formed. The hydroperoxide peak will first increase, and then decrease, as hydroperoxides 
are decomposed to form crosslinked species. The intensity of the peaks, related to these crosslinked species, increases over time. First, the dimers are visible, then trimers, and, given enough reaction time, higher oligomers are detected.

\section{Determination of the hydroperoxide concentration in ethyl linoleate}

During storage, the hydroperoxide concentration in EL will slowly increase because of the spontaneous reaction with dioxygen from the air. Since hydroperoxides have a direct influence on the metal-catalyzed autoxidation, in most cases it is desirable to remove all of the hydroperoxides from EL prior to use in an oxidation experiment. In the remainder of this paper, whenever 'purified EL' is mentioned, it implies that EL is used, from which all peroxides have been removed.

Hydroperoxides are removed from EL by eluting it over a basic $\mathrm{Al}_{2} \mathrm{O}_{3}$ column. To determine the hydroperoxide content in EL before and after purification, the HPLC method described by Nakamura and Maeda was used. ${ }^{21}$ This method is based on the stoichiometric oxidation of triphenylphosphine (TP) to triphenylphosphine oxide (TPO) by any hydroperoxides that are present in a given sample. The concentration of formed TPO can then be determined by reverse phase HPLC using an UV detector at $220 \mathrm{~nm}$. All hydroperoxides are quantitatively reduced within $30 \mathrm{~min}$, and the detection limit for TPO at $220 \mathrm{~nm}$ is lower than 10 pmol. Hydroperoxides are also important intermediates in the autoxidation reaction. Therefore, it is valuable to determine the hydroperoxide level in an autoxidation reaction mixture at different reaction times. For this purpose, a spectrophotometric method was used. ${ }^{22}$

\section{Performance of the commercial driers}

\section{FT-IR experiments: comparison of different commercial catalysts}

It appeared to be possible to compare the drying ability of different metal complexes in the model system by using time-resolved FT-IR. In Fig. 7, the resulting firstorder fits for three different drying agents are compared. ${ }^{3}$

The commercial drier cobalt 2-ethylhexanoate (Co-EH) and the drier based on manganese 2-ethylhexanoate and bipyridine (Mn-EH/bpy) both show excellent catalytic activity in the disappearance of EL (reaction rate $30-200 \mathrm{~min}: 0.004 \mathrm{~min}^{-1}$ ). For the $\mathrm{Mn}-\mathrm{EH} / \mathrm{bpy}$ system, a short induction time of $20 \mathrm{~min}$ is observed. Mn-EH (manganese 2-ethylhexanoate), in contrast, is not active in the test system during the first $100 \mathrm{~min}$. After this induction time, the Mn-EH is also active in the reaction, with a slightly lower reaction

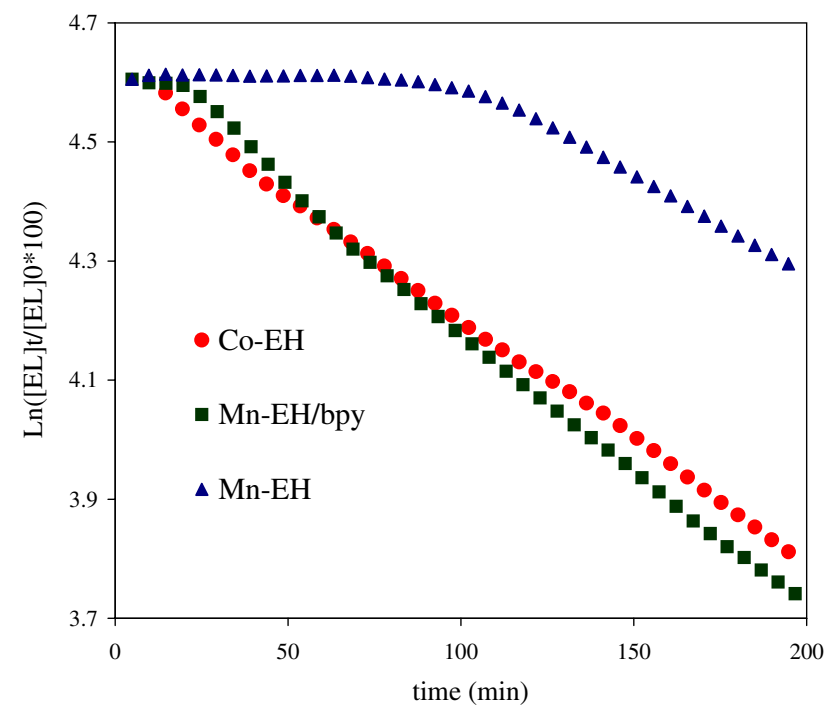

Fig. 7: FT-IR graphs representing the pseudo first-order rates for the disappearance of EL in time for the catalytic driers Co Nuodex (O), Mn-EH/bpy ( $\square$ ), and Mn-EH (A)

rate of $0.0032 \mathrm{~min}^{-1}$. These results are in reasonable correlation with the drying times found for actual alkyd paints. ${ }^{3}$

\section{Influence of bipyridine}

The remarkable enhancing effect of bpy on the catalytic activity of the manganese octoate in the drier $\mathrm{Mn}-\mathrm{EH} / \mathrm{bpy}$ has been explored in a systematic study. Known amounts of bpy were added to Mn-EH, and these in situ mixtures were tested in the model reaction. For comparison, the effect of bipyridine on the catalytic activity of Co-EH was also investigated. ${ }^{3}$

The addition of only 0.25 equiv. of bipyridine to Mn-EH already has a tremendous effect on the catalytic activity. The induction time drops from 100 to $25 \mathrm{~min}$, and the slope of the graph increases from $3.2 \times 10^{-3}$ to $4.4 \times 10^{-3}$. By further increasing the bpy/manganese ratio, the induction time can be reduced to $10 \mathrm{~min}$, and the slope of the graph stays the same within the experimental error. The in situ measurements of $\mathrm{Co}-\mathrm{EH}$ with various amounts of bpy show quite a different trend. Here, the induction time remains short in every measurement. The slope, however, decreases significantly with increasing amounts of added bipyridine. The resulting graphs resemble the measurements taken with lower Co concentrations as measured for the determination of the optimal catalyst ratio. It can be assumed that by adding bpy, a number of the cobalt ions is co-ordinated, thereby lowering the concentration of the catalytically active species.

During the studies, single crystals of the tetranuclear manganese cluster $\left[\mathrm{Mn}_{4} \mathrm{O}_{2}\right.$ (2-ethylhexanoate) ${ }_{6}(\text { bpy })_{2}$ ] were obtained. ${ }^{3} \mathrm{~A}$ projection of the structure is depicted in Fig. 8. Due to a disorder in the alkyl 


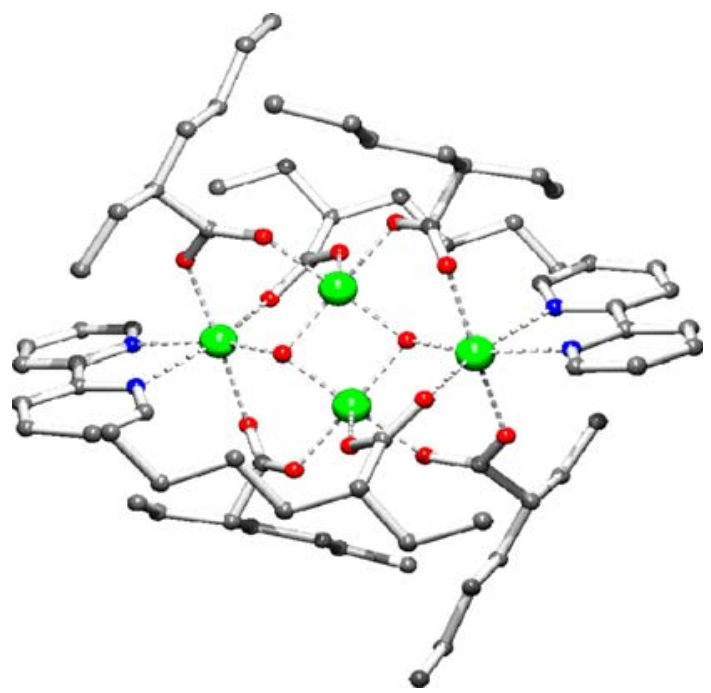

Fig. 8: Molecular structure of $\left[\mathrm{Mn}_{4} \mathrm{O}_{2}\right.$ (2-ethylhexanoate) ${ }_{6}$ $(\text { bpy })_{2}$ ]

chains of the 2-ethylhexanoate, overlaid by strongly disordered free solvent molecules on the same location and twinning of the crystals, the structure could not be fully refined. The overall structure, however, especially the geometry of the $\mathrm{Mn}_{4} \mathrm{O}_{2}$ cluster core, can be considered as quite fitting. A very similar structure of an acetate-bridged cluster has been published by Vincent et al. ${ }^{23}$ The cluster was found to contain a mixed-valence complex with two of the manganese ions in the Mn(III)oxidation state in a penta-coordinated pure oxygen environment. The two other manganese ions are octahedrally co-ordinated in a $\mathrm{N}_{2} \mathrm{O}_{4}$ donor set with much longer bonding distances, and these are in the divalent oxidation state. Since there is a strong analogy between the geometry in the central $\mathrm{Mn}_{4} \mathrm{O}_{2}$ core, it is assumed that in the 2-ethylhexanoate-bridged complex the electronic distribution is the same. In the FT-IR experiments with EL, this cluster shows only a short induction time and a slope that is even slightly higher than those of the in situ measurements, indicating that this tetranuclear species may be the actual catalyst in the EL oxidation.

\section{Development of new driers based on manganese ${ }^{2,19}$}

\section{FT-IR measurements}

The FT-IR plots of selected experiments are depicted in Fig. 9, and the autoxidation rates and induction times of $\mathrm{Mn}-\mathrm{EH}, \mathrm{Co}-\mathrm{EH},\left[\mathrm{Mn}(\mathrm{acac})_{3}\right],\left[\mathrm{Mn}(\mathrm{acac})_{3}\right] /$ bpy and $\left[\mathrm{Mn}(\mathrm{acac})_{2}(\mathrm{bpy})\right]$ are listed in Table 1. Large variations in induction times are observed when unpurified EL is used as the substrate. Unpurified EL contains hydroperoxides in varying amounts because of slow air oxidation during storage. The presence of

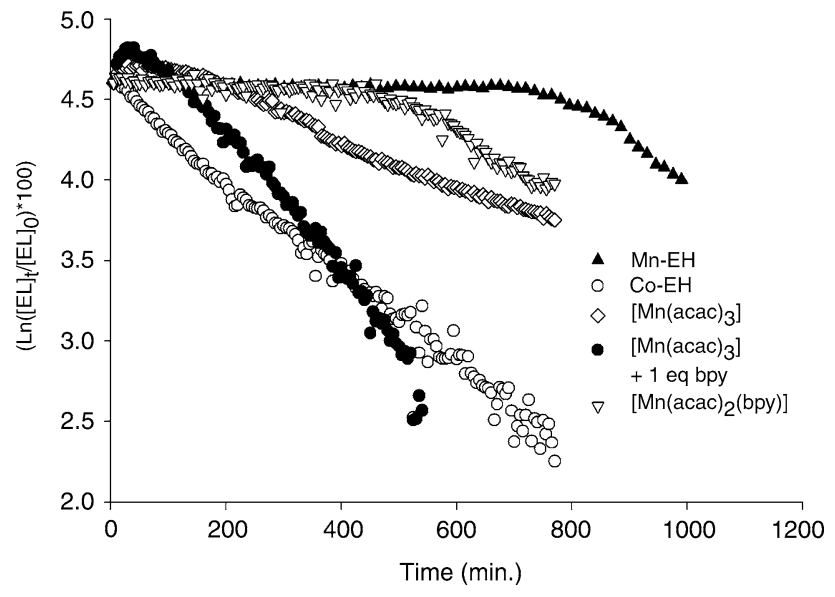

Fig. 9: Time-dependent integral plots of the $3010 \mathrm{~cm}^{-1}$ IR peak of purified EL reaction with different catalysts

hydroperoxides directly influences the reaction rate and the induction time. When purified EL is used, the induction time for the commercial manganese catalyst Mn-EH increases from 140 to over $700 \mathrm{~min}$. Short induction times (varying between 0 and $100 \mathrm{~min}$ ) are observed, however, when manganese(III) acetylacetonate is used as an oxidation catalyst. The higher oxidation activity for $\left[\mathrm{Mn}(\mathrm{acac})_{3}\right]$ is compared with that of Mn-EH can be attributed mostly to the large induction time for $\mathrm{Mn}-\mathrm{EH}$. When the EL oxidation rate for $\left[\mathrm{Mn}(\mathrm{acac})_{3}\right]$ is compared with the rates for $\mathrm{Mn}$ $\mathrm{EH}$ and $\mathrm{Co}-\mathrm{EH}$, it is clear that although $\left[\mathrm{Mn}(\mathrm{acac})_{3}\right]$ shows a slightly lower rate than Co-EH, its overall activity is far superior to that of Mn-EH. The addition of 1 equiv. of bipyridine to a reaction mixture of $\left[\mathrm{Mn}(\mathrm{acac})_{3}\right]$ and $\mathrm{EL}$ increases the rate of oxidation of EL significantly, surpassing even the activity of the cobalt drier. Adding more than 1 equiv., however, does not seem to enhance the rate any further.

To investigate the influence of bipyridine in more detail, the complex $\left[\mathrm{Mn}^{\mathrm{II}}(\mathrm{acac})_{2}\right.$ (bpy)] was synthesized

Table 1: Oxidation rates and induction times for the oxidation of $\mathrm{EL}^{\mathrm{a}}$

Induction Rate constant $k$ time (min) $\quad\left(10^{-3} \mathrm{~min}^{-1}\right)$

\begin{tabular}{lrr}
\hline Co-EH & 0 & 3 \\
$\mathrm{Mn}-\mathrm{EH}$ & $>700$ & 2 \\
{$\left[\mathrm{Mn}^{\prime \prime \prime}(\mathrm{acac})_{3}\right]$} & $<100$ & 1 \\
{$\left[\mathrm{Mn}^{\prime \prime \prime}(\mathrm{acac})_{3}\right]+1$ equiv. bpy } & 30 & 4 \\
{$\left[\mathrm{Mn}^{\prime \prime \prime}(\mathrm{acac})_{3}\right]+2$ equiv. bpy } & 50 & 4 \\
{$\left[\mathrm{Mn}^{\prime \prime \prime}(\mathrm{acac})_{3}\right]+3$ equiv. bpy } & 45 & 5 \\
{$\left[\mathrm{Mn}^{\prime \prime}(\mathrm{acac})_{2}(\mathrm{bpy})\right]$} & 430 & 2
\end{tabular}

${ }^{a} \mathrm{EL}$ was purified by eluting over $\mathrm{Al}_{2} \mathrm{O}_{3}$ before use. Molar ratio catalyst $/ E L=1 / 400$, reaction in neat $E L$ at room temperature. Due to the radical nature of the reactions the average experimental error is about $20 \%$ 
separately, ${ }^{24}$ and was tested for its autoxidation activity. A rather large induction time is observed when this manganese(II) compound is used in combination with purified EL (see Table 1). Initiation does not occur with manganese(II) complexes in the absence of peroxides because manganese(II) is not able to oxidize the substrate under these reaction conditions. Thus, an induction time of over $400 \mathrm{~min}$ is observed in which either the substrate or the complex is slowly airoxidized, after which the catalytic radical autoxidation can start. To check whether or not the induction time results from the absence of peroxides, the oxidation experiment was also performed with unpurified EL. Then no induction time is observed and the oxidation reaction starts immediately with a rate of $4 \times 10^{-3} \mathrm{~min}^{-1}$, comparable to the rates observed for $\left[\mathrm{Mn}(\mathrm{acac})_{3}\right]$ with bpy added in situ.

\section{Structure of $\left[\mathrm{Mn}(\mathrm{acac})_{2}(\mathrm{bpy})\right]^{24}$}

While searching for novel manganese-based catalysts for the oxidative drying of alkyd paints, the X-ray structure of the well-known compound $\left[\mathrm{Mn}(\mathrm{acac})_{2}\right.$ (bpy)] was determined. Although it has been claimed that this complex has an octahedral co-ordination geometry ${ }^{25}$ it appears to be the first example of a mixed-ligand complex with innocent didentate ligands that possesses the trigonal prismatic co-ordination geometry.

A projection of the complex is shown in Fig. 10. The manganese(II) ion has a almost perfect $\mathrm{N}_{2} \mathrm{O}_{4}$ trigonalprismatic co-ordination environment, with two acetylacetonate ligands and one bipyridine ligand. The manganese to nitrogen distances are 2.288(3) and 2.283(2) $\AA$. The manganese to oxygen distance for the acetylacetonate ligands is, for each $\mathrm{Mn}-\mathrm{O}$ bond, almost the same, and lie in the range $2.1480(16)$ to $2.1580(18) \AA$. The bite angles for each of the acetylacetonate ligands are also nearly identical, being $81.69(6)^{\circ}(\mathrm{O} 1-\mathrm{Mn} 1-\mathrm{O} 2)$ and $81.92(6)^{\circ}(\mathrm{O} 3-\mathrm{Mn} 1-\mathrm{O} 4)$. Since the bite angles are rather small and the $\mathrm{Mn}-\mathrm{O}$

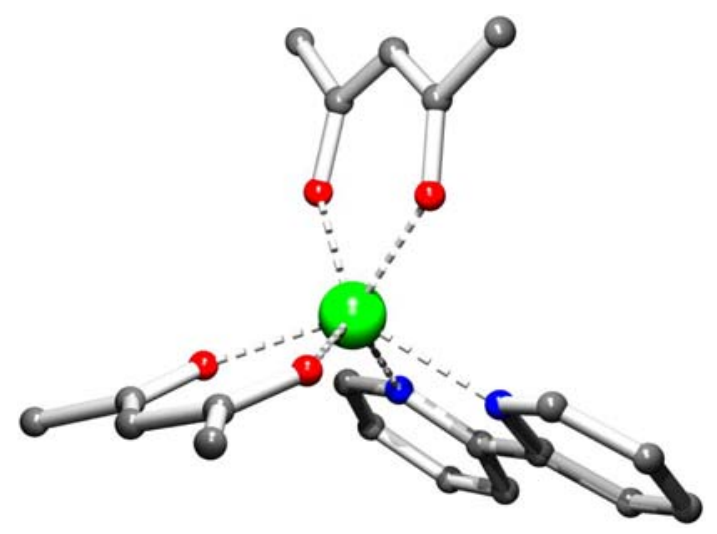

Fig. 10: Projection of $\left[\mathrm{Mn}(\mathrm{acac})_{2}(\mathrm{bpy})\right]$. Hydrogen atoms were omitted for clarity

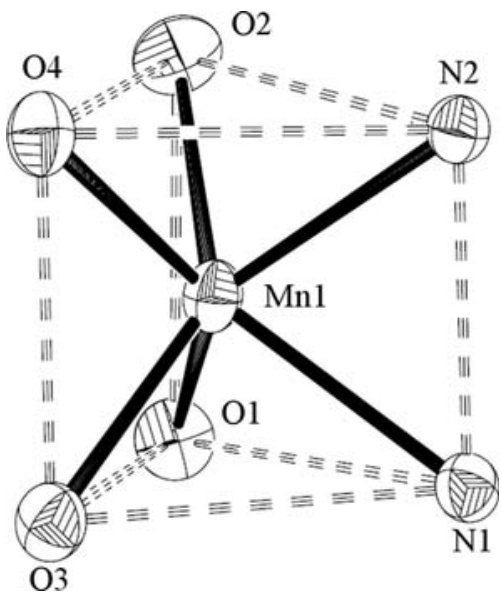

Fig. 11: Trigonal prismatic geometry of $\left[\mathrm{Mn}(\mathrm{acac})_{2}(\mathrm{bpy})\right]$ in detail

distance rather large, the $\mathrm{Mn}-\mathrm{O}-\mathrm{C}$ angles are also large, in the range of $130.39(17)$ to $131.78(16)^{\circ}$. The bipyridine ligand has a bite angle (N1-Mn1-N2) of $70.41^{\circ}$. The two acetylacetonate ligands are nearly planar, but the angle between the least-square planes through the rings of the bipyridine ligand amounts to $3.79(12)^{\circ}$. This ligand is therefore not planar. For all three ligands, the atoms comprising the chelate rings deviate little from their least-square mean plane. The obtuse angles between the least-square mean planes of the chelate rings lie in the range of 117.67(7) to $121.30(6)^{\circ}$, in accordance with the trigonal prismatic co-ordination geometry.

The two trigonal faces of the prism constitute one oxygen atom of each of the acetylacetonate ligands and one nitrogen atom, thus forming $\mathrm{O} 1-\mathrm{O} 3-\mathrm{N} 1$ and O2-O4-N2. In Fig. 11 the trigonal prismatic coordination geometry around manganese is shown in more detail. The lengths of the triangular sides are in the range of 2.901(2) to 3.030(3) $\AA$ for the triangle O1-O3-N1, and 2.846(2) to 3.007(3) $\AA$ for the triangle O2-O4-N2. All of the angles are in the range of $56.88(7)$ to $62.25(7)^{\circ}$. The four acetylacetonate oxygen atoms make up an almost exact square, the sides of which are in the range of 2.816(2) to 2.901(2) $\AA$. The remaining two faces of the prism are trapezoids consisting of two oxygen atoms of one acetylacetonate ligand, and are joined by the two nitrogen atoms of the bipyridine. Density functional theory (DFT) calculations have been performed to address the question of the preference for trigonal prismatic vs octahedral geometry, comparing this complex with the related octahedral phenanthroline complex, [Mn(acac $)_{2}($ phen $\left.)\right]$.

\section{The time-evolution of peroxides in EL oxidation}

The concentration of peroxides was monitored over time for the autoxidation of EL in the presence of the various catalysts. The results are shown in Fig. 12. The peroxide value for the unreacted EL, as received from 


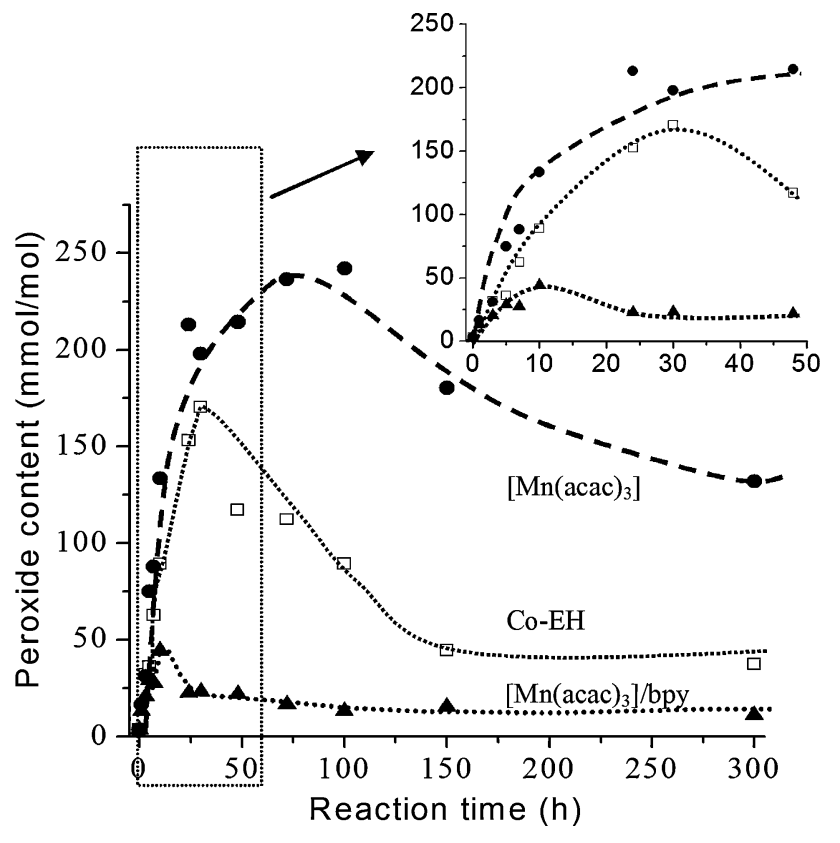

Fig. 12: The evolution of peroxide amounts during the oxidation of EL in the presence of various catalysts. Lines are added to aid the eye

the supplier, was determined to be $4 \mathrm{mmol} / \mathrm{mol} \mathrm{EL}$. In the presence of $\mathrm{Co}-\mathrm{EH}$, a peroxide concentration was reached of about $50 \mathrm{mmol} / \mathrm{mol}$ in $5 \mathrm{~h}$. As the oxidation of EL proceeds, the amount of peroxides increases continuously and a maximum value of $170 \mathrm{mmol} / \mathrm{mol}$ is reached in about $30 \mathrm{~h}$. Then, the peroxide value decreases rapidly to a level of approximately $40 \mathrm{mmol} / \mathrm{mol}$. These observations are in accordance with EL-autoxidation studies reported by Mallegol et al. ${ }^{26,27}$

In the presence of $\left[\mathrm{Mn}(\mathrm{acac})_{3}\right]$, a much higher concentration of peroxides $(240 \mathrm{mmol} / \mathrm{mol})$ is reached after a much longer reaction time of $100 \mathrm{~h}$, and the rate-of-decrease of the peroxide value after attaining the maximum value is much less than for the oxidation with Co-EH. Even after 300 h, a significant amount of peroxide of $130 \mathrm{mmol} / \mathrm{mol}$ still remains.

When bpy is added to $\left[\mathrm{Mn}(\mathrm{acac})_{3}\right]$, a totally different observation can be made. The concentration of peroxides is, at all times, much lower than is observed for the other two cases: a maximum value is reached of about $45 \mathrm{mmol} / \mathrm{mol}$ in just $10 \mathrm{~h}$ of reaction time. The amount of peroxide then decreases to a very low level of $10 \mathrm{mmol} / \mathrm{mol}$ over the course of the experiment (up to $300 \mathrm{~h}$ ).

\section{Oligomerization during EL oxidation}

The oxidation reactions of EL generally lead to the formation of oligomers, which is pivotal for the drying and hardening of real alkyd coatings. In Fig. 13, the SEC chromatograms are shown of EL after various

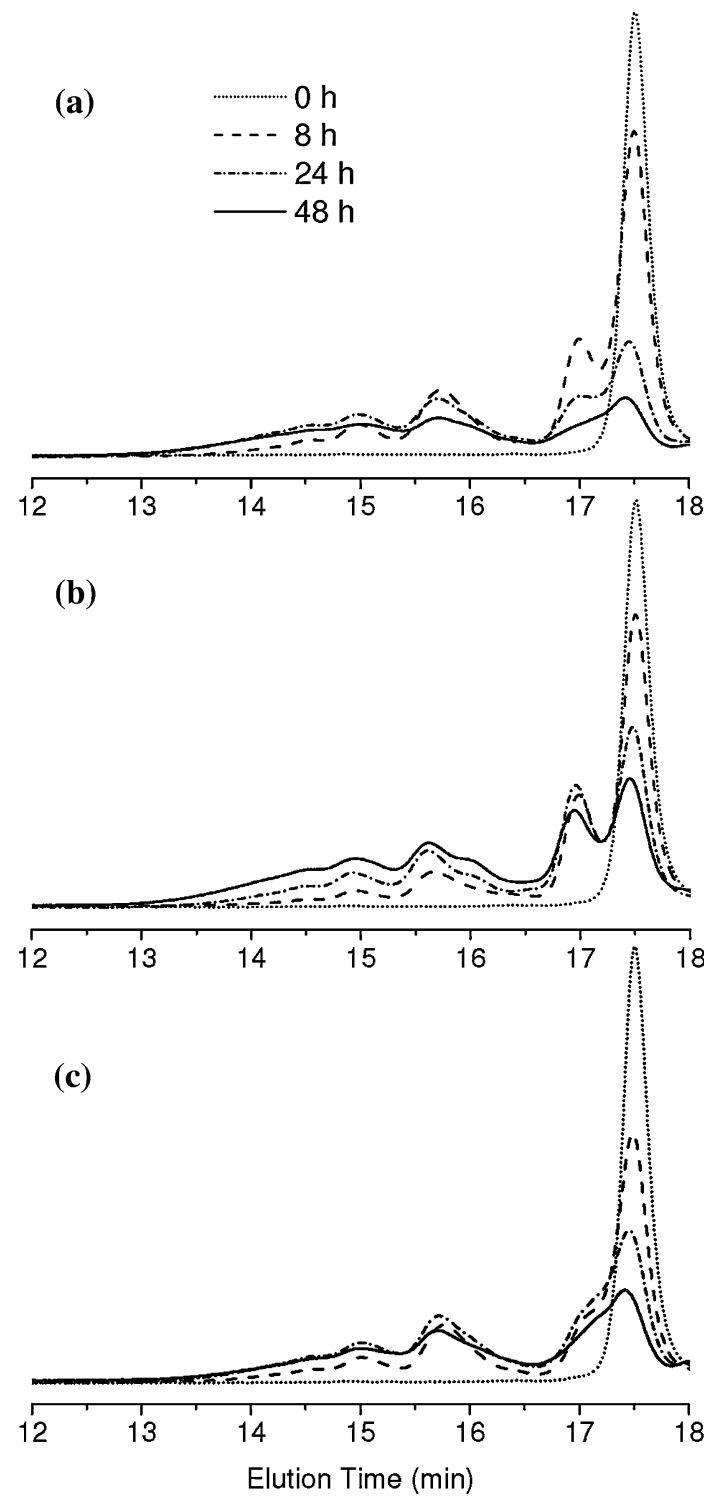

Fig. 13: SEC of EL after different periods of oxidation in the presence of various catalysts: (a) Co-EH, (b) [Mn(acac) $)_{3}$, and (c) $\left[\mathrm{Mn}(\mathrm{acac})_{3}\right] / \mathrm{bpy}$

periods of oxidation catalyzed by $\mathrm{Co}-\mathrm{EH},\left[\mathrm{Mn}(\mathrm{acac})_{3}\right]$, and $\left[\mathrm{Mn}(\mathrm{acac})_{3}\right] / \mathrm{bpy}$ (see Fig. 6 for assignments). After oxidation of EL for $8 \mathrm{~h}$ catalyzed by Co-EH, dimers, trimers, and higher oligomers could be readily observed. As the oxidation proceeds for $24 \mathrm{~h}$, more trimers and higher oligomers are formed as the dimer peak slightly decreases. ${ }^{13}$ After $48 \mathrm{~h}$, both the dimer and trimer peaks have decreased relative to their levels after $24 \mathrm{~h}$ because of the formation of higher oligomers, and possibly because of the oxidative degradation of the fatty acid chains via $\beta$-scission reactions. The EL peak does not disappear completely even after $48 \mathrm{~h}$, which is because of the presence of about $8 \%$ of nonreactive saturated esters and the presence of $19 \%$ of the less reactive ethyl oleate $e^{13,28}$ in the technical-grade 
EL. The peak appearing at $17 \mathrm{~min}$ is due to EL hydroperoxides $3,4,12$ and to other oxygen-containing EL derivatives (for example, epoxide species). ${ }^{12}$ The amount of hydroperoxides first increases and then decreases significantly over the course of the reaction, in agreement with the results obtained for the peroxide value determination.

In the oxidation of EL with $\left[\mathrm{Mn}(\mathrm{acac})_{3}\right]$, the same observations can be made after $8 \mathrm{~h}$ as for the oxidation with Co-EH. Dimers and trimers are formed, although to a lesser extent than for the Co-EH catalyzed reaction. In contrast to the oxidation with $\mathrm{Co}-\mathrm{EH}$, however, the amount of (higher) oligomers only increases with prolonged reaction times, indicating little degradation of the oligomerized polymer network, in agreement with the head-space GC-MS results discussed below, which showed less hexanal and pentanal formation for $\left[\mathrm{Mn}(\mathrm{acac})_{3}\right]$ in comparison with the other catalysts. The peak due to hydroperoxides remains significant for the entire duration of the experiment $(48 \mathrm{~h})$, again in agreement with the results obtained for the peroxide value determination for the oxidation with $\left[\mathrm{Mn}(\mathrm{acac})_{3}\right]$. The peak due to the EL-monomer also remains considerable, which was in perfect agreement with the difference in the EL oxidation rates previously found for $\mathrm{Co}-\mathrm{EH}$ and $\left[\mathrm{Mn}(\mathrm{acac})_{3}\right]$ using FT-IR.

In the oligomerization of EL with $\left[\mathrm{Mn}(\mathrm{acac})_{3}\right] / \mathrm{bpy}$, the same trends in the formation of the dimer, trimer, and higher oligomers are seen as for the oxidation with Co-EH. Also, for this catalyst, a decrease in the dimer and trimer peaks is observed over time, which can, in part, be attributed to the degradation of the polymer network, as was clear from the GC-MS results discussed below. The peroxide peak is rather low in intensity at all times, in accordance with the observations made in the peroxide value determination (see Fig. 12).

\section{Formation of volatile byproducts during EL oxidation}

The characteristic odor of air-drying alkyd paints is due to the formation of volatile aldehydes, typically hexanal and pentanal. These aldehydes are formed through $\beta$-scission reactions of alkoxy radicals. ${ }^{10}$ The amount of hexanal and pentanal formed during the oxidation of EL in the presence of different catalysts has been quantified by head-space GC-MS, and the results are shown in Fig. 14. In the reaction that was catalyzed by Co-EH, the amount of hexanal and pentanal increases steadily over the course of the reaction $(105 \mathrm{~h})$, reaching levels of 16.6 and $0.4 \mathrm{mmol} /$ mol EL, respectively. During the first $50 \mathrm{~h}$ of the oxidation with the $\left[\mathrm{Mn}(\mathrm{acac})_{3}\right] / \mathrm{bpy}$ system, much more hexanal is generated than when Co-EH is used (see Fig. 11a). However, after $100 \mathrm{~h}$, the hexanal levels for both catalysts are at a similar level. The amount of pentanal that is generated with the $\left[\mathrm{Mn}(\mathrm{acac})_{3}\right] / \mathrm{bpy}$ catalyst is significantly higher. In the oxidation with only $\left[\mathrm{Mn}(\mathrm{acac})_{3}\right]$ as a catalyst, lower amounts of both hexanal and pentanal are formed compared with the reactions with $\mathrm{Co}-\mathrm{EH}$ or $\left[\mathrm{Mn}(\mathrm{acac})_{3}\right] / \mathrm{bpy}$.

\section{Cyclic voltammetry}

In order to compare the oxidizing power of the complex $\left[\mathrm{Mn}(\mathrm{acac})_{3}\right]$ with and without bipyridine, cyclic voltammetry has been used. ${ }^{2}$ The cyclic voltammograms of $\left[\mathrm{Mn}(\mathrm{acac})_{3}\right]$ and $\left[\mathrm{Mn}(\mathrm{acac})_{2}(\mathrm{bpy})\right]$ are shown in Fig. 15. The Mn(II)/Mn(III) couple (peaks $1 / 1^{\prime}$ and $\left.\mathrm{a} / \mathrm{a}^{\prime}\right)$ shifts towards a more positive potential for $\left[\mathrm{Mn}(\mathrm{acac})_{2}(\mathrm{bpy})\right]$ compared with $\left[\mathrm{Mn}(\mathrm{acac})_{3}\right]$. The voltammograms of $\left[\mathrm{Mn}(\mathrm{acac})_{2}(\mathrm{bpy})\right]$, obtained at different scan rates, show that the relative intensity of peak $a^{\prime}$ increases with the increasing scan rate. This indicates that the species $\left[\mathrm{Mn}^{\mathrm{III}}(\mathrm{acac})_{2}(\mathrm{bpy})\right]^{+}$is unstable under these conditions and therefore it is likely that the more stable $\left[\mathrm{Mn}(\mathrm{acac})_{3}\right]$ complex is formed. Peak b results from the oxidation of $\left[\mathrm{Mn}(\mathrm{acac})_{3}\right]$ and peak $\mathrm{b}^{\prime}$ from the reduction of $\left[\mathrm{Mn}^{\mathrm{IV}}(\mathrm{acac})_{3}\right]^{+}$. Indeed, the fact that the $\mathrm{Mn}(\mathrm{III}) /(\mathrm{IV})$ transitions in the voltammograms (peaks 2,2' and $b / b^{\prime}$ ) occur nearly at the same potentials suggests also that they must both arise from the same species (i.e. $\left.\left[\mathrm{Mn}(\mathrm{acac})_{3}\right]\right)$. Peak c most probably results from the reduction of $\left[\mathrm{Mn}^{\mathrm{III}}(\mathrm{acac})_{3}\right]$ to $\left[\mathrm{Mn}^{\mathrm{II}}(\mathrm{acac})_{3}\right]^{-}$, which can be concluded by comparison with peak $1^{\prime}$. The reduced complex $\left[\mathrm{Mn}^{\mathrm{II}}(\mathrm{acac})_{3}\right]^{-}$
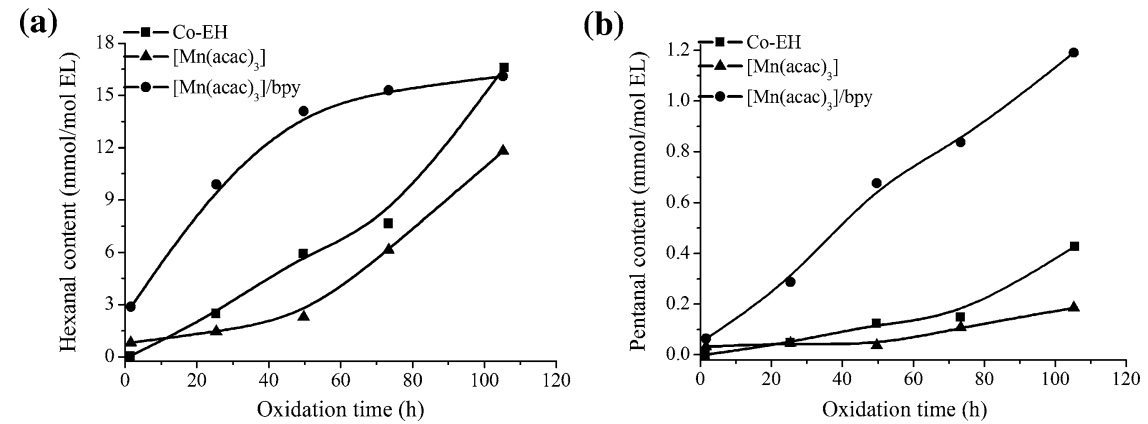

Fig. 14: The formation of volatile byproducts (a) hexanal and (b) pentanal during the oxidation of EL in the presence of different catalysts 


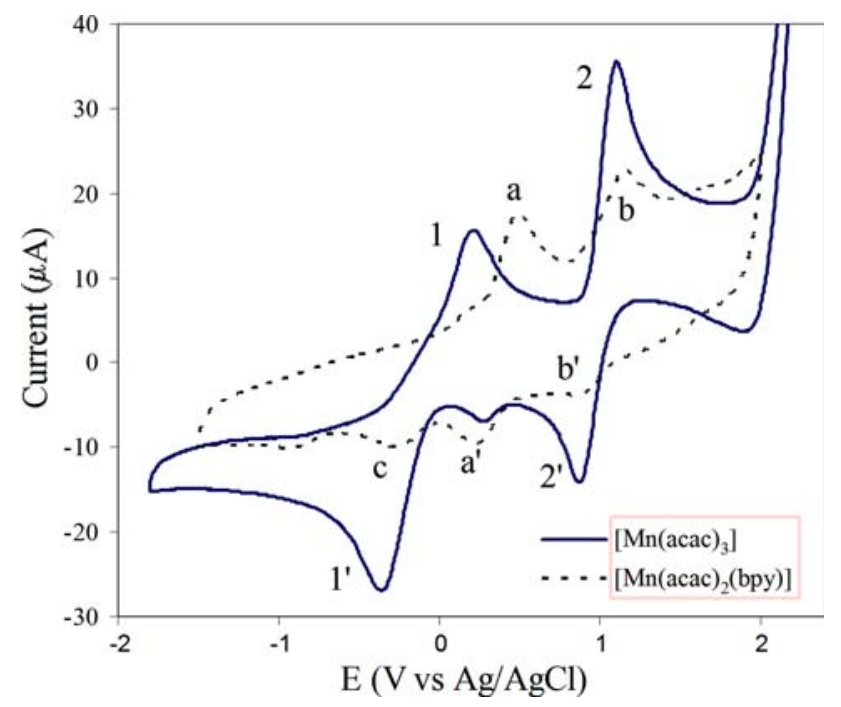

Fig. 15: Cyclic voltammograms of [Mn(acac) $\left.)_{3}\right]$ and [Mn (acac) $)_{2}$ (bpy)] in $\mathrm{CH}_{3} \mathrm{CN},\left[{ }^{n} \mathrm{Bu}_{4} \mathrm{~N}\right]\left(\mathrm{PF}_{6}\right)$ as electrolyte, scan rate $200 \mathrm{mV} / \mathrm{s}$ selected potentials: $1 / 1^{\prime} 0.222 /-0.373 \mathrm{~V}$; a/a' $0.50 / 0.203 \mathrm{~V} ; 2 / 2^{\prime} 1.111 / 0.862 \mathrm{~V} ; b^{\prime} b^{\prime} 1.165 / 0.891 \mathrm{~V} ; \mathrm{c}-0.28 \mathrm{~V}$

reacts with bpy in solution to form the more stable complex $\left[\mathrm{Mn}^{\Pi}(\mathrm{acac})_{2}(\mathrm{bpy})\right]$, which is supported by the absence of a (re)oxidation peak for $\left[\mathrm{Mn}^{\mathrm{II}}(\mathrm{acac})_{3}\right]^{-}$. The cyclic voltammogram of an in situ mixture of $\left[\mathrm{Mn}(\mathrm{acac})_{3}\right.$ ] with bpy is nearly identical to that of $\left[\mathrm{Mn}(\mathrm{acac})_{2}(\mathrm{bpy})\right]$, confirming the instability of the complexes and the occurrence of rapid equilibrium reactions.

\section{Experiments in real alkyd paint}

The complex $\left[\mathrm{Mn}(\mathrm{acac})_{3}\right]$ was also tested as a drier in a real alkyd paint formulation, both in the absence and in the presence of bpy. The drying time of the paint was determined using two methods that are common in the paint industry: using the 'thumb-test' and the use of a Braive recorder. ${ }^{29,30}$ The drying times for $\left[\mathrm{Mn}(\mathrm{acac})_{3}\right]$ and $\left[\mathrm{Mn}(\mathrm{acac})_{3}\right] / \mathrm{bpy}$ and the results obtained for the commercial combination of cobalt driers and manganese driers are listed in Table 2. From the results presented in Table 2, it is clear that $\left[\mathrm{Mn}(\mathrm{acac})_{3}\right]$ and $\left[\mathrm{Mn}(\mathrm{acac})_{3}\right] /$ bpy have paint-drying activities which are considerably better than the activities of the standard manganese paint driers because the total drying times were halved for the new driers. The drying results, compared with the drying times obtained with the cobalt driers, are comparable or even slightly better. It is also interesting to note that the drying times for $\left[\mathrm{Mn}(\mathrm{acac})_{3}\right]$ appear to be even shorter than that for the $\left[\mathrm{Mn}(\mathrm{acac})_{3}\right] /$ bpy system.

\section{Discussion}

\section{Radical initiation by [Mn(acac $\left.)_{3}\right]$}

Mn(III) is well known as a radical initiator, mostly in the form of $\left[\mathrm{Mn}(\mathrm{OAc})_{3}\right]$ in acetic acid, at elevated temperatures and often in the presence of radical-chain starters such as NHPI ( $N$-hydroxyphthalimide). ${ }^{31,32}$ $\left[\mathrm{Mn}(\mathrm{acac})_{3}\right]$, in combination with co-catalysts such as ${\text { acetic } \text { acid }^{33,34} \text { or benzyl bromide }}^{35}$ is also known to act as a radical initiator in the radical polymerization of various substrates.

The induction time in the oxidation of EL is a measure of the ability of the catalyst to initiate the autoxidation radical-chain reaction. Consequently, from the short induction times observed in the FT-IR measurements, it seems that $\left[\mathrm{Mn}(\mathrm{acac})_{3}\right]$ is able to initiate the radical autoxidation of EL directly and at room temperature. Initiation therefore occurs even in the absence of any acids, halides, peroxides or other easily oxidizable radical-chain starters. A tentative mechanism for the initiation reaction is depicted in Fig. 16. Radical initiation is proposed to occur via a hydrogen atom abstraction pathway, in analogy with a mechanism for the oxidation of 1,4-cyclohexadiene by tris(hexafluoroacetylacetonato)-manganese(III) as described by Bryant et al. ${ }^{36} \mathrm{H}$-atom transfer from EL to $\left[\mathrm{Mn}^{\mathrm{III}}(\mathrm{acac})_{3}\right]$ results in the formation of $\left[\mathrm{Mn}^{\mathrm{II}}\right.$ $\left.(\text { acac })_{2}\right]$, a free acetylacetone ligand and an ethyl

Table 2: Drying times of real alkyd paint, using different catalysts ${ }^{a}$

Drier

Drying time by hand

Braive recorder drying time

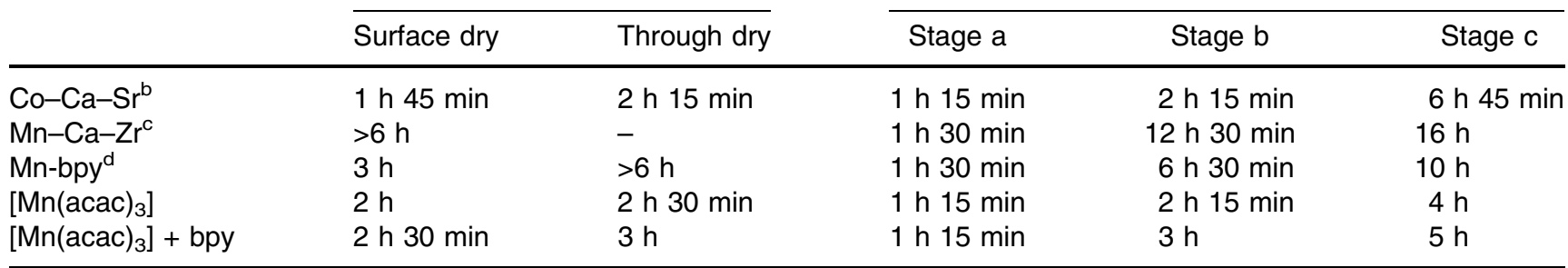

a The drying time was measured in a clear varnish based on Uralac AD 152 WS-40 from DSM Resins (a medium oil alkyd resin based on soy-bean oil, $47 \%$ oil)

b Commercial cobalt combination drier

c Commercial manganese combination drier

d Commercial manganese drier based on manganese 2-ethylhexanoate and bipyridine 


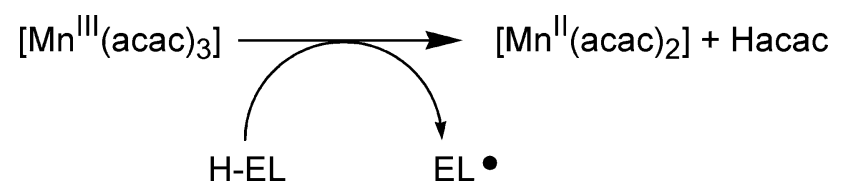

Fig. 16: Radical initiation by [Mn(acac) $\left.)_{3}\right]$ through hydrogen atom abstraction of the activated methylene group in ethyl linoleate

linoleate radical $\left(\mathrm{EL}^{\bullet}\right)$. This ethyl linoleate radical reacts immediately with dioxygen $\left(\mathrm{O}_{2}\right)$ to form a peroxy radical, which can form a hydroperoxide by further abstraction of an $\mathrm{H}^{\bullet}$ from another EL molecule.

\section{Oxidation of EL}

The FT-IR results show that $\left[\mathrm{Mn}(\mathrm{acac})_{3}\right]$ catalyzes the oxidation of EL at a reasonable rate, although this rate is lower than that of the oxidation with Co-EH. From the data relating to the amounts of peroxide, it becomes clear that the high oxidation rate for the $\left[\mathrm{Mn}(\mathrm{acac})_{3}\right] /$ bpy system might be attributed to an extremely efficient capability of hydroperoxide decomposition.

In Fig. 17, a set of radical reactions is given that are often proposed for hydroperoxide (ROOH) build-up in metal-catalyzed autoxidation reactions. ${ }^{37}$ The hydroperoxide concentration, at a given time, depends on the ratio of the rates for hydroperoxide decomposition vs hydroperoxide formation. ${ }^{38}$ According to the reactions depicted in Fig. 17, the higher the rate for reactions $\mathbf{4}$ and $\mathbf{5}$, the lower the hydroperoxide concentration will be. It appears that the [Mn $\left.(\mathrm{acac})_{3}\right] /$ bpy catalyst is able to decompose $\mathrm{ROOH}$ almost as soon as it is formed, thus attaining a very low ROOH concentration. In Fig. 18, a tentative overview is given for the metal-catalyzed peroxide decomposition reactions for each catalyst used in the present study.

The cobalt catalyst is mainly a robust peroxide decomposition catalyst, where the rate for reaction 7 will probably be comparable with the rate for reaction 8. It is clear from Fig. 12, in which it is shown that the $\mathrm{ROOH}$ concentration decreases steadily after the maximum has been reached, that the cobalt catalyst retains its decomposition activity for at least $150 \mathrm{~h}$. The oxygen uptake data for the Co-EH-catalyzed EL oxidation show that significant over-oxidation of EL takes place (oxygen uptake is higher than $1 \mathrm{~mol}$ $\mathrm{O}_{2} /$ mol EL). The most common pathways for overoxidation are: (1) through radical addition to conjugated double bond systems, generating a new carbon-centered radical which can again react with dioxygen, ${ }^{15}$ or (2) via oxidation of the products that are generated by a $\beta$-scission reaction of an alkoxyl radical.

Reactions $\mathbf{9}$ and $\mathbf{1 0}$ given in Fig. 18 are the proposed $\mathrm{ROOH}$ decomposition reactions for the catalyst $\left[\mathrm{Mn}(\mathrm{acac})_{3}\right]$. Additionally, the direct activation of EL via reaction 11 also plays a role for $\left[\mathrm{Mn}(\mathrm{acac})_{3}\right]$, as shown in Fig. 16. The most important difference for the activity of $\left[\mathrm{Mn}(\mathrm{acac})_{3}\right]$ compared with $\mathrm{Co}-\mathrm{EH}$ is the rate with which reactions $\mathbf{9}$ and especially $\mathbf{1 0}$ proceed (apparent from the Raman data and the rate for oxygen uptake). A lower rate for these reactions can explain the significantly higher hydroperoxide concentration that is attained through reactions $\mathbf{2}$ and $\mathbf{3}$ in Fig. 17. Additional hydroperoxides can be formed through reaction $\mathbf{1 1}$ in Fig. 18.

As previously mentioned, the $\left[\mathrm{Mn}(\mathrm{acac})_{3}\right] / \mathrm{bpy}$ catalyst shows a very high rate for the oxidation of EL. The high oxidation activity is ascribed to the in situ formation of the compound $\left[\mathrm{Mn}^{\mathrm{II}}(\mathrm{acac})_{2}(\mathrm{bpy})\right]$ through the sequence of reactions 9 and $\mathbf{1 2}$ (or $\mathbf{1 1}$ and 12). The species $\left[\mathrm{Mn}^{\mathrm{II}}(\mathrm{acac})_{2}(\mathrm{bpy})\right]$ has most probably a very high rate for reaction 13, forming species $\left[\mathrm{Mn}^{\mathrm{III}}(\mathrm{acac})_{2}(\mathrm{bpy})\right]^{+}$. The cyclic voltammetry measurements have shown that this complex has a reduction wave at $0.203 \mathrm{~V}$ (vs $\mathrm{Ag} / \mathrm{AgCl}$ in $\mathrm{CH}_{3} \mathrm{CN}$ ). This makes the species $\left[\mathrm{Mn}^{\mathrm{III}}(\mathrm{acac})_{2}(\mathrm{bpy})\right]^{+}$a much more potent oxidizing agent than, for example, $\left[\mathrm{Mn}^{\mathrm{III}}(\mathrm{acac})_{3}\right]$, which has the corresponding reduction wave at $-0.373 \mathrm{~V}$ (vs $\mathrm{Ag} / \mathrm{AgCl}$ in $\mathrm{CH}_{3} \mathrm{CN}$ ). The reduction of $\mathrm{Mn}$ (III) is always the slower reaction in Haber-Weiss reactions. ${ }^{31}$ The addition of bpy thus effectively 'de-bottlenecks' the autoxidation reaction by speeding up the slower step. Reactions 13, 14, and $\mathbf{1 5}$ shown in Fig. 18 will thus proceed at a much higher rate than reactions $\mathbf{9 , 1 0}$, and $\mathbf{1 1}$. The extremely high rate of dioxygen uptake, coupled to the very low levels of detected hydroperoxides, may be explained by the assumption that the oxidation will most likely be dominated by reactions $\mathbf{1 3}$ and $\mathbf{1 5}$, once the species $\left[\mathrm{Mn}^{\mathrm{II}}(\mathrm{acac})_{2}(\mathrm{bpy})\right]$ is formed. The alkoxy radicals formed (see reaction 13) have a high tendency to undergo $\beta$-scission, thus forming hexanal and pentanal. The formation of alkoxy radicals disrupts the radical

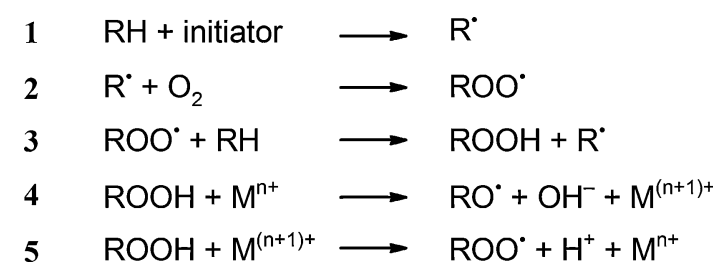

Fig. 17: General metal-catalyzed (M) autoxidation reactions, which are responsible for hydroperoxide (ROOH) build-up and decomposition 

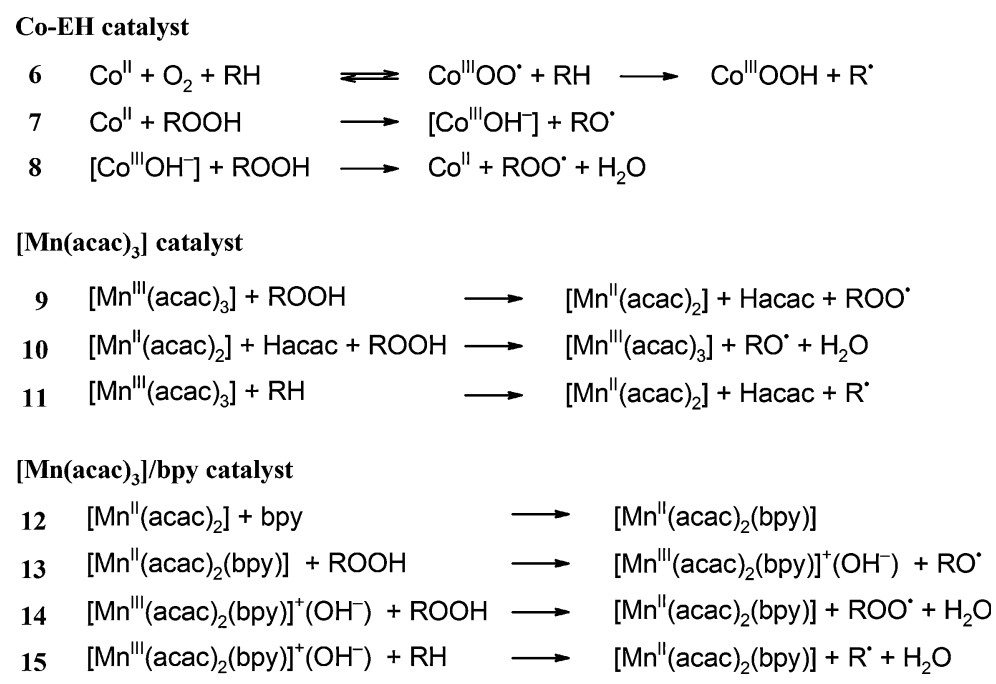

Fig. 18: Proposed sets of reactions for each of the catalyst systems

chain of reactions 2 and 3 (see Fig. 17), and consequently the build-up of a high ROOH concentration. An explanation for the complete cessation of the uptake of oxygen, once all hydroperoxides are decomposed, could be that the $\left[\mathrm{Mn}^{\mathrm{III}}(\mathrm{acac})_{2}(\mathrm{bpy})\right]^{+}$complex can only rapidly abstract a doubly allylic hydrogen atom. Once each molecule of EL has reacted with at least one molecule of dioxygen, no doubly allylic hydrogen atoms and hydroperoxides are available (because the hydroperoxides are rapidly decomposed) and hence reactions 13,14 , and 15 will stop.

\section{Oligomerization of EL}

A major difference between the SEC results for $\mathrm{Co}-\mathrm{EH}$ and that for $\left[\mathrm{Mn}(\mathrm{acac})_{3}\right]$ is the amount of oligomers that are formed over a period of $48 \mathrm{~h}$. The levels of dimer, trimer, and higher oligomers are clearly greater for the $\left[\mathrm{Mn}(\mathrm{acac})_{3}\right]$ catalyst. This observation can be explained by the high ROOH level that is generated through the cycle of reactions $\mathbf{2}, \mathbf{3}$, and 11. Each hydroperoxide group that is formed results in the formation of a set of conjugated double bonds. A high $\mathrm{ROOH}$ concentration therefore implies a high concentration of conjugated double bonds that are especially prone to radical addition reactions, because of the possibility of forming a relatively stable allylic radical. ${ }^{15}$ Consequently, each time an alkoxy, or especially a peroxo radical species, is formed through decomposition of a hydroperoxide, a probable reaction pathway for the radical is to react with a conjugated double bond to form a higher oligomer. Another reason for the lower level of oligomerization, as observed for $\mathrm{Co}-\mathrm{EH}$, is degradation of the polymer network that is formed. The head-space GC-MS data for the Co-EH-catalyzed EL oxidation reaction show that constant hexanal and pentanal formation takes place, a sure sign of $\beta$-scission reactions. For $[\mathrm{Mn}$ $\left.(\mathrm{acac})_{3}\right]$, the amounts of hexanal and pentanal that are formed are lower, but the same trend is followed, as previously observed for the autoxidation of EL catalyzed by Co-EH. This observation supports the assumption that a lower rate for the ROOH decomposition reactions is probably the most prominent difference between the oxidation mechanisms by which $\left[\mathrm{Mn}(\mathrm{acac})_{3}\right]$ and Co-EH function.

The SEC chromatogram for the oxidation of EL, catalyzed by $\left[\mathrm{Mn}(\mathrm{acac})_{3}\right] / \mathrm{bpy}$, is similar to that for the Co-EH catalyzed EL oxidation, the exception being the low intensity of the hydroperoxide-peak (in agreement with the results for the hydroperoxide determination). As discussed in the previous section, the $\left[\mathrm{Mn}(\mathrm{acac})_{3}\right] /$ bpy catalyst will probably predominantly operate through reactions $\mathbf{1 3}$ and $\mathbf{1 5}$ and thus will generate a higher level of alkoxy radicals relative to the other catalysts. The high amounts of hexanal and pentanal that are formed compared with those with $\mathrm{Co}-\mathrm{EH}$ and $\left[\mathrm{Mn}(\mathrm{acac})_{3}\right]$ are in agreement with this assumption. A system that predominantly generates alkoxy radicals will have a lower extent of oligomerization because these radicals generally have more pathways to form other products of low molecular weights. $^{10}$

\section{The best paint drier}

The cobalt(II) 2-ethylhexanoate catalyst is the most widely used oxidative drier for alkyd paints. Judging only by the oxidation rate of the alkyd model compound EL, it is tempting to say that the manganese catalyst system $\left[\mathrm{Mn}(\mathrm{acac})_{3}\right] /$ bpy would be an ideal replacement for the cobalt catalyst as a drier. The results pertaining to the formation of higher oligomers show, however, that the slower catalyst $\left[\mathrm{Mn}(\mathrm{acac})_{3}\right]$ 
yields a much greater extent of oligomerization. This observation is supported by the drying results in actual alkyd paint formulations, where the catalyst $\left[\mathrm{Mn}(\mathrm{acac})_{3}\right]$ shows slightly better drying performance than does the system $\left[\mathrm{Mn}(\mathrm{acac})_{3}\right] / \mathrm{bpy}$. In real alkyd systems, cobalt complexes are never used as the only drier: so-called 'secondary' driers are added, sometimes used to retard the activity of the cobalt drier. ${ }^{39}$ The lesser performance of $\left[\mathrm{Mn}(\mathrm{acac})_{3}\right] / \mathrm{bpy}$ in real alkyd paints (see Table 2) might indeed be attributed to the fact that it is too active: the autoxidation reactions yield predominantly alkoxy radicals, which result in less oligomerization. Thus, although the $\left[\mathrm{Mn}(\mathrm{acac})_{3}\right]$ catalyst shows a lower rate of oxidation in our EL test system, as a paint drier it is the better candidate to replace the cobalt catalyst systems.

\section{Conclusions}

It has become clear that the oxidation reaction of EL, as monitored with time-resolved FT-IR spectroscopy, may well be suitable as a high-throughput screening model reaction for new catalysts. The rates of disappearance of EL in the reaction with dioxygen, as catalyzed by the commercial cobalt driers and the manganese driers, correlate reasonably well with the actual drying times as found in the real alkyd paints. The method seems to be quite sensitive to changes in the catalyst's structure, which is shown by the various results obtained with different amounts of bipyridine added to Mn-EH or Co-EH.

Some early insight into the active catalytic species in the drying of alkyd paints has been gained. The presence of the tetranuclear cluster $\left[\mathrm{Mn}_{4} \mathrm{O}_{2}\right.$ (2-ethylhexanoate $)_{6}(\mathrm{bpy})_{2}$ ] in the commercial $\mathrm{Mn}-\mathrm{EH} / \mathrm{bpy}$ drier and its catalytic activity in the oxidation of EL, gives a strong indication that this type of cluster may play an important role in catalytic oxidative drying. The compound $\left[\mathrm{Mn}(\mathrm{acac})_{3}\right]$ is an efficient catalyst for the oxidation and the oligomerization of EL, which is proposed to proceed not only via hydroperoxide decomposition, but also through substrate activation. $\left[\mathrm{Mn}(\mathrm{acac})_{3}\right]$ also functions as a very good drying catalyst in a real alkyd system, better than conventional manganese driers and comparable with, or even better than a cobalt combination drier. The system $\left[\mathrm{Mn}(\mathrm{acac})_{3}\right]$ with added bpy has a very high activity for the oxidation of EL. The in situ formation of the species $\left[\mathrm{Mn}^{\mathrm{II}}(\mathrm{acac})_{2}(\mathrm{bpy})\right]$ and $\left[\mathrm{Mn}^{\mathrm{III}}(\mathrm{acac})_{2}(\mathrm{bpy})\right]^{+}$ and the high reactivities of these two species with $\mathrm{ROOH}$ and EL, respectively, are proposed as an explanation for the observed high oxidation rate. In the oxidation of EL by the $\left[\mathrm{Mn}(\mathrm{acac})_{3}\right] /$ bpy catalyst, less oligomerization and a greater amount of volatile products were observed, probably due to the generation of predominantly alkoxy radicals in hydroperoxide decomposition. In real alkyd systems, adding bpy to $\left[\mathrm{Mn}(\mathrm{acac})_{3}\right]$ is therefore not advantageous. This is also evident from the longer drying times that have been observed for $\left[\mathrm{Mn}(\mathrm{acac})_{3}\right] /$ bpy in a real alkyd system when compared with the drying times for $\left[\mathrm{Mn}(\mathrm{acac})_{3}\right]$.

In subsequent studies, the presented new model reaction can be applied to screen large numbers of manganese complexes and iron complexes for their potential as drying catalysts. Since of the continuing shift of solventborne alkyds to water-based systems, attention has also been directed to the role of water in our model reaction. ${ }^{7}$

Acknowledgments These investigations have been supported by the Netherlands Research Council for Chemical Sciences (CW) with financial aid from the Netherlands Technology Foundation (STW) in the Priority Programme Materials and by the Innovation Oriented Research Programme on Heavy Metals (IOP Zware Metalen, no IZW99241c), sponsored by the Netherlands Ministry of Economic Affairs.

The authors are grateful to Dr ST Warzeska, Dr J-C Hierso, Dr S Grecea, Dr J-Z Wu, Dr ZO Oyman (Technical University Eindhoven), and Dr W Ming (Technical University Eindhoven) for their work and collaboration on this topic. The authors thank Dr J Bieleman (Elementis, Delden), Dr WJ Muizebelt, and Dr W Buijs (DSM, Geleen) for fruitful discussions. $\mathrm{We}$ are indebted to $\mathrm{Mr} \mathbf{J}$ ter Borg (Elementis, Delden) for the determination of the drying time in alkyd paints. The authors are grateful to $\mathrm{Dr} \mathrm{H}$ Kooijman (Utrecht University) for the X-ray diffraction studies.

\section{References}

1. van Gorkum, R, Bouwman, E, "The Oxidative Drying of Alkyd Paint Catalysed by Metal Complexes." Coord. Chem. Rev., 249 1709-1728 (2005)

2. van Gorkum, R, Bouwman, E, Reedijk, J, "Fast Autoxidation of Ethyl Linoleate Catalyzed by [Mn(acac)3] and Bipyridine: A Possible Drying Catalyst for Alkyd Paints." Inorg. Chem., 43 (8) 2456-2458 (2004)

3. Warzeska, ST, Zonneveld, M, van Gorkum, R, Muizebelt, WJ, Bouwman, E, Reedijk, J, “The Influence of Bipyridine on the Drying of Alkyd Paints: A Model Study." Prog. Org. Coat., 44 (3) 243-248 (2002)

4. Tanase, S, Bouwman, E, Reedijk, J, "Role of Additives in Cobalt-mediated Oxidative Cross-linking of Alkyd Resins." Appl. Catal. A-Gen., 259 (1) 101-107 (2004)

5. Tanase, S, Bouwman, E, Reedijk, J, Driessen, WL, Ferbinteanu, M, Huber, M, Mills, AM, Spek, AL, "Synthesis and Structural Studies of Two New Base Adducts of Bis(2,4Pentanedionato)Cobalt(II)." Eur. J. Inorg. Chem. 1963-1969 (2004)

6. Tanase, S, Hierso, JC, Bouwman, E, Reedijk, J, ter Borg, J, Bieleman, JH, Schut, A, "New Insights on the Anti-Skinning Effect of Methyl Ethyl Ketoxime in Alkyd Paints." New J. Chem., 27 (5) 854-859 (2003)

7. Wu, J-Z, Bouwman, E, Reedijk, J, "Chelating Ligands as Powerful Additives to Manganese Driers for Solvent-borne and Water-borne Alkyd Paints." Prog. Org. Coat., 49 103108 (2004) 
8. Oyman, ZO, Ming, W, Micciché, F, Oostveen, E, van Haveren, J, van der Linde, R, "A Promising Environmentally-friendly Manganese-based Catalyst for Alkyd Emulsion Coatings." Polymer, 45 (22) 7431-7436 (2004)

9. Frankel, EN, Garwood, RF, Vinson, JR, Weedon, BCL, "Stereochemistry of Olefin and Fatty-acid Oxidation. 1. Autoxidation of Hexene and Hepta-2,5-diene Isomers." $J$. Chem. Soc. - Perkin. Trans. I., 2707-2713 (1982)

10. Hubert, JC, Venderbosch, RAM, Muizebelt, WJ, Klaasen, RP, Zabel, KH, "Mechanistic Study of Drying of Alkyd Resins Using (Z,Z)- and (E,E)-3,6-Nonadiene as Model Substances." Prog. Org. Coat., 31 (4) 331-340 (1997)

11. Carless, HAJ, Batten, RJ, "Photosensitised Oxidation of Model Unsaturated Lipid Systems-(4z,7z)-Undeca-4,7-diene and (4z)-Undec-4-en-7-yne." J. Chem. Soc. - Perkin. Trans. I., 1999-2007 (1987)

12. Muizebelt, WJ, Donkerbroek, JJ, Nielen, MWF, Hussem, JB, Biemond, MEF, Klaasen, RP, Zabel, KH, "Oxidative Crosslinking of Alkyd resins Studied with Mass Spectrometry and NMR Using Model Compounds.' J. Coat. Technol., 70 (876) 83-93 (1998)

13. Muizebelt, WJ, Hubert, JC, Venderbosch, RAM, "Mechanistic Study of Drying of Alkyd Resins Using Ethyl Linoleate as a Model Substance." Prog. Org. Coat., 24 (1-4) 263-279 (1994)

14. Falla, NAR, "Linoleic-based Coatings - A Study of the Dry Film Structure." J. Coat. Technol., 64 (815) 55-60 (1992)

15. Muizebelt, WJ, Nielen, MWF, "Oxidative Cross-linking of Unsaturated Fatty Acids Studied with Mass Spectrometry." J. Mass Spectrometry, 31 (5) 545-554 (1996)

16. Hartshorn, JH, "Time-lapse Infrared Spectroscopic Investigation of Alkyd and Linseed Oil Cure." J. Coat. Technol., 54 (687) 53-61 (1982)

17. Vandevoort, FR, Ismail, AA, Sedman, J, Emo, G, "Monitoring the Oxidation of Edible Oils by Fourier-transform Infrared-spectroscopy." J. Am. Oil Chem. Soc., 71 (3) 243253 (1994)

18. Muizebelt, WJ, Hubert, JC, Nielen, MWF, Klaasen, RP, Zabel, KH, "Cross-link Mechanisms of High-solids Alkyd Resins in the Presence of Reactive Diluents." Prog. Org. Coat., 40 (1-4) 121-130 (2000)

19. Oyman, ZO, Ming, W, van der Linde, R, van Gorkum, R, Bouwman, E, "Effect of $\left[\mathrm{Mn}(\mathrm{acac})_{3}\right]$ and its Combination with 2,2'-Bipyridine on the Autoxidation and Oligomerisation of Ethyl Linoleate." Polymer, 46 1731-1738 (2005)

20. Socrates, G, Infrared Characteristic Group Frequencies. Wiley \& Sons, New York (1980), ISBN: 0471275921

21. Nakamura, T, Maeda, H, "A Simple Assay for Lipid Hydroperoxides based on Triphenylphosphine Oxidation and High-performance Liquid-chromatography." Lipids, 26 (9), 765-768 (1991)

22. Jiang, ZY, Hunt, JV, Wolff, SP, "Ferrous Ion Oxidation in the Presence of Xylenol Orange for Detection of Lipid Hydroperoxide in Low-density-lipoprotein." Anal. Biochem., 202 (2) 384-389 (1992)

23. Vincent, JB, Christmas, C, Chang, HR, Li, QY, Boyd, PDW, Huffman, JC, Hendrickson, DN, Christou, G, "Modeling the
Photosynthetic Water Oxidation Center - Preparation and Properties of Tetranuclear Manganese Complexes Containing $\mathrm{Mn}_{4} \mathrm{O}_{2}$ 6+,7+,8+ Cores and the Crystal-structures of $\mathrm{Mn}_{4} \mathrm{O}_{2}\left(\mathrm{O}_{2} \mathrm{CMe}\right)_{6}(\mathrm{Bipy})_{2}$ and $\mathrm{Mn}_{4} \mathrm{O}_{2}\left(\mathrm{O}_{2} \mathrm{CMe}\right)_{7}(\mathrm{Bipy})_{2}$ (ClO $)$ )." J. Am. Chem. Soc., 111 (6) 2086-2097 (1989)

24. van Gorkum, R, Buda, F, Kooijman, H, Spek, AL, Bouwman, E, Reedijk, J, "Trigonal Prismatic vs Octahedral Geometry for Mn(II) Complexes with Innocent Didentate Ligands: A Subtle Difference as Shown by XRD and DFT on [Mn (acac) $)_{2}$ (bpy)]." Eur. J. Inorg. Chem. 2255-2261 (2005)

25. Percy, GC, Thornton, DA, "Crystal Field Aspects of Vibrational Spectra. 4. Effect of d-orbital Splitting on Infrared Spectra of Transition Metal(II) Complexes of cisChelating Bidentate Ligands." J. Mol. Struct., 10 (1) 39 (1971)

26. Mallegol, J, Lemaire, J, Gardette, JL, “Drier Influence on the Curing of Linseed Oil.” Prog. Org. Coat., 39 (2-4) 107$113(2000)$

27. Mallegol, J, Gonon, L, Commereuc, S, Verney, V, "Thermal (DSC) and Chemical (Iodometric titration) Methods for Peroxide Measurements in Order to Monitor Drying Extent of Alkyd Resins." Prog. Org. Coat., 41 (1-3) 171-176 (2001)

28. Frankel, EN, "Lipid Oxidation - Mechanisms, Products and Biological Significance." J. Am. Oil Chem. Soc., 61 (12) 1908-1917 (1984)

29. Ali, M, McWhinnie, WR, "Organobismuth(III) and Organobismuth(V) Carboxylates and their Evaluation as Paint Driers." App. Organomet. Chem., 7 (2) 137-141 (1993)

30. van Gorkum, R, Bouwman, E, Reedijk, J, EP1382648 (2004)

31. Sheldon, RA, Kochi, JK, In: Eley, DD, Pines, H, Weisz, PB (eds) Metal Catalyzed Oxidations of Organic Compounds in the Liquid Phase: A Mechanistic Approach, pp. 274-414. Academic Press, New York (1976) ISBN: 0120078252

32. Snider, BB, "Manganese(III)-based Oxidative Free-radical Cyclizations." Chem. Rev., 96 (1) 339-363 (1996)

33. Lavrov, NA, "Copolymerisation of $N$-vinylsuccinimide with Vinyl-acetate in Aqueous-solution, Initiated by the Manganese Tris(acetylacetonate)-acetic Acid System." Russ. J. Appl. Chem., 67 (9) 1358-1360 (1994)

34. Lavrov, NA, "Kinetic Features of Polymerization of 2-Hydroxyethyl Methacrylate, Initiated by the System Manganese Tris(acetylacetonate)-acetic Acid." Russ. J. Appl. Chem., 68 (6) 922-924 (1995)

35. Endo, K, Yachi, A, "Molecular-weight-controlled Polymerisation of Styrene with $\mathrm{Mn}(\mathrm{acac})_{3}$ in Combination with Organic Halides.” Polym. Bull., 46 (5) 363-369 (2001)

36. Bryant, JR, Taves, JE, Mayer, JM, "Oxidations of Hydrocarbons by Manganese(III) Tris(hexafluoroacetylacetonate)." Inorg. Chem., 41 (10) 2769-2776 (2002)

37. Frankel, EN, Lipid Oxidation. The Oily Press Ltd., Dundee, Scotland (1998) ISBN: 0951417193

38. Reich, L, Stivala, S, Autoxidation of Hydrocarbons and Polyolefins. Marcel Dekker Inc, New York (1969)

39. Middlemiss, RG, Olszanski, DJ, "New Catalysts for Highsolids Coatings, Part 1." Am. Paint Coat. J., 78 35-43 (1993) 